\title{
Prevalence of Depression and Psychological Distress and Perturbations of Cortisol Dynamics in Attendants of Hospitalized Patients: An Observational Pilot Study
}

\author{
Rimesh Pal ${ }^{1}$, Naresh Sachdeva ${ }^{1}$, Anil Bhansali ${ }^{1}$, Akhilesh Sharma ${ }^{2}$, Rama Walia ${ }^{1}$ \\ 1. Endocrinology, Post Graduate Institute of Medical Education \& Research, Chandigarh, IND 2. Psychiatry, Post \\ Graduate Institute of Medical Education \& Research, Chandigarh, IND
}

Corresponding author: Rama Walia, ramawaliapgimer@gmail.com

\begin{abstract}
Background: Attendants of in-hospital patients are prone to undue stress resulting in depression, anxiety, melancholy and psychological distress. Hitherto available studies cater to attendants of patients admitted in critical care units and none have ventured to look into their cortisol dynamics. Herein, we have evaluated the magnitude of psychological distress and depression amongst ostensibly healthy attendants of noncritically ill patients and correlated them with cortisol dynamics.

Methods: Non-critically ill patients admitted to the general medicine ward were chosen by purposive sampling and one attendant was selected from each patient. Those with known risk factors, psychiatric illnesses, chronic drug intake, addictions, and overweight/obesity were excluded. Psychological distress and depression were assessed using the General Health Questionnaire 12-items (GHQ-12) and the Patient Health Questionnaire (PHQ-9), respectively. Morning plasma cortisol, late-night plasma cortisol (LNPC), late-night salivary cortisol (LNSC), urinary free cortisol (UFC), and plasma cortisol after overnight $1 \mathrm{mg}$ dexamethasone administration were measured.

Results: After exclusion, 39 participants were recruited (M:F=2.3:1.6). The mean age was $34.1 \pm 11.4$ years. The mean duration of stay in hospital ambience prior to recruitment was $16.2 \pm 1.2$ days. Based on the PHQ-9 score, $55 \%$ of the participants had depression. Psychological distress prevailed in $13 \%$ of participants as per the GHQ-12 score. The median LNPC/LNSC was higher in participants with depression compared to those with no depression, however, there was no statistically significant difference. There was no significant correlation between GHQ-12/PHQ-9 scores and cortisol dynamics.
\end{abstract}

Conclusions: Although depression is prevalent in about half of the patient attendants, cortisol dynamics remain largely unaltered over a short period of two to three weeks.

Review began $11 / 26 / 2020$ Review ended 12/06/2020 Published 12/13/2020

(c) Copyright 2020 Pal et al. This is an open access article distributed under the terms of the Creative Commons Attribution License CC-BY 4.0., which permits unrestricted use, distribution, and reproduction in any medium, provided the original author and source are credited.
Categories: Endocrinology/Diabetes/Metabolism, Internal Medicine, Psychiatry

Keywords: ghq-12, patient attendant, psychological distress, depression, cortisol dynamics, phq-9

\section{Introduction}

Patients' attendants are an indispensable adjunct to the hospital staff. They work just as much as the healthcare professionals that expose them to significant occupational stress, which culminates in psychological disorders (depression, anxiety), fatigue, dissatisfaction, and maladaptive behaviors (substance abuse) [1]. However, an individual's personality and coping skills ultimately decide whether and to what extent hospital ambience will lead to psychological distress. In general, females are more prone to develop psychological disorders than males [2], mostly because of the prevalent social barriers.

Psychological health among attendants of in-hospital patients has never been investigated in detail. Few available studies have mostly concentrated on family members and relatives of patients admitted in the intensive care unit (ICU) and similar critical care settings [3-10]. All those studies have unanimously reiterated that family members of critically ill patients are fraught with stress, anxiety, depression, melancholy, and post-traumatic stress disorder (PTSD). However, studies performed in critical care settings might not reflect the actual psychological status in non-critical scenarios. 'Fear of impending death', one of the principal stressors in critical care settings might not be of that significant relevance in non-critical situations.

Disturbances in the pituitary-hypothalamic-adrenal axis are common in chronic psychological disorders [11]. Cortisol excess is the most common anomaly, being reported in as many as $40-60 \%$ of drug-naive patients of major depression [12]. Consequently, patients of melancholic depression suffer from numerous somatic sequelae that include osteoporosis, metabolic syndrome, coronary artery disease, and certain infectious and 
neoplastic diseases [13]. The other spectrum includes hypocortisolism that has been reported in 20-25\% of patients with stress-related disorders like chronic fatigue syndrome, atypical depression, burn-out, and PTSD [14]. Altered cortisol dynamics have also been observed in professions associated with undue stress like night-shift workers and nursing staff [15-17]. However, abnormalities in the cortisol axis have hitherto never been studied amongst attendants of hospitalized patients.

Hence, the present study was undertaken to evaluate the prevalence of psychological distress amongst apparently healthy attendants of patients admitted to the general ward of a tertiary care institution and correlate with various aspects of cortisol dynamics.

\section{Materials And Methods}

A prospective observational study was conducted at the 120-bedded general medicine ward of a tertiary care government hospital in Northern India for over three months. Non-critically ill patients admitted in the ward were chosen every four weeks by systematic random sampling selecting every fifth bed from a random starting point. Thereafter, one family member/relative was selected from each chosen patient by simple random sampling using a random number table. Inclusion criteria included age more than 18 years, literate (able to read and write in Hindi), no prior history of any comorbid conditions with particular emphasis on diabetes mellitus, hypertension, coronary artery disease, connective tissue disease, rheumatological disease, bronchial asthma, chronic obstructive pulmonary disease, chronic liver or kidney disease or any known psychiatric disorder, no history of any chronic drug intake, no history of any addictions and body mass index (BMI) of $18.5-22.9 \mathrm{~kg} / \mathrm{m} 2$. The participant must have been attending to his/her index patient in the hospital ward for at least two weeks before being included in the study. A baseline history was elicited from all the eligible participants to exclude any pre-existing psychiatric disorder. Written informed consent was obtained from all the participants. The study was approved by the Institute Ethics Committee, Post Graduate Institute of Medical Education and Research, India.

\section{Psychological health questionnaires}

Psychological distress and depression were evaluated with well-validated questionnaires a couple of days before assessing cortisol dynamics. The primary investigator thoroughly explained the questionnaires and then the participants were asked to fill them up by themselves.

Psychological distress was measured using the General Health Questionnaire short-form 12-items (GHQ12) [18]. The standardized Hindi version was used for participant feasibility [19]. Each item was measured on a four-point Likert scale (one=better than usual, four=much worse than usual). As recommended, items were binary recoded (1-2=0, 3-4=1) before being summed. Individuals with scores of 7 and below were considered 'probable normal', scores from 8 to 15 as 'probable psychological distress' and more than 15 as 'definite psychological distress' [19].

Depression was assessed with the patient health questionnaire depression module (PHQ-9). It has been well validated in the general population and has been found to be useful in detecting major and subclinical depressive disorders [20]. The PHQ-9 scores each of the 9 Diagnostic and Statistical Manual of Mental Disorders, Fourth Edition (DSM-IV) criteria as '0' (not at all) to '3' (nearly every day). As for GHQ-12, the Hindi version of PHQ-9 was used. All nine individual scores were summed up to get the final score. A total score of 0-4 was regarded as having 'no depression', 5-9 as 'mild depression', 10-14 as 'moderate depression', and 15-19 as 'moderately severe depression'.

\section{Assessment of cortisol dynamics}

Every participant had the following parameters measured as a part of a comprehensive assessment of cortisol dynamics: (a) Morning plasma cortisol, (b) late-night plasma cortisol (LNPC), (c) 24 hours urinary free cortisol (UFC), (d) late-night salivary cortisol (LNSC), and (e) 1-mg overnight dexamethasone suppression test (ONDST).

\section{Method of blood sample collection for the estimation of cortisol}

A morning plasma cortisol sample was collected exactly between 0800-0900 hours. For late-night plasma cortisol, a blood sample was drawn at 2300 hours in the awake state. All the blood samples were centrifuged immediately, plasma separated and stored at $-20^{\circ} \mathrm{C}$. Samples were transported to the laboratory in an ice pack for processing. ONDST was performed by orally administering a 1-mg tablet of dexamethasone at 2300 hours with a blood sample for cortisol collected at 0800 hours the next day.

\section{Method of saliva collection for the estimation of LNSC}

On the day of sample collection, participants were instructed to eat at least two hours prior to collection and not to brush their teeth for at least four hours prior to the test. Salivate (SalivaBio Oral Swab from Salimetrics, Carlsbad, CA) was given and patients were asked to moisten it with saliva by keeping it in their mouth for three to five minutes. Saliva was then collected in a plain vial at 2300 hours. Samples were kept in a refrigerator and transported to the laboratory the next day. 


\section{Method of urine collection for the estimation of 24 hours UFC}

Urine was collected for 24 hours for three consecutive days. Participants were instructed to void urine at 0900 hours on the first day of collection and collect all the urine in a clean container until the next morning at 0900 hours and store it in the ward refrigerator. The total volume was measured and $30 \mathrm{~mL}$ of sample was sent to the laboratory for cortisol estimation. All three samples were stored at $-20^{\circ} \mathrm{C}$ for subsequent processing. The average of the three values was considered as the 24 hours UFC of the participant. It was ensured that ONDST be performed after completion of urine collection as prior administration of dexamethasone could lead to suppression of the hypothalamic-pituitary-adrenal axis and falsely low UFC values.

\section{Assays used}

Plasma and salivary cortisol were measured using electrochemiluminescence immunoassay (ECLIA) (ELECSYS-2010, Roche Diagnostics, Basel, Switzerland). UFC was measured using the dichloromethane extraction method. Inter- and intra-assay coefficients of variation were $1.4-2.8 \%, 1.5-14.2 \%$, and $1.8-4.7 \%$ for plasma cortisol, LNSC, and UFC, respectively.

The cut-offs used to define hypercortisolism were based on a study performed by Jarial et al. in 24 patients of Cushing's syndrome and 16 patients of pseudo-Cushing's states [21]. Values that had the maximum sensitivity in diagnosing hypercortisolism were considered. Accordingly, a cut-off of $213.67 \mathrm{nmol} / \mathrm{L}$ for morning plasma cortisol and $116.04 \mathrm{nmol} / \mathrm{L}$ for LNPC was chosen (sensitivity being $100 \%$ for each). LNSC and 24 hours UFC had $100 \%$ sensitivity at a value of $4.95 \mathrm{nmol} / \mathrm{L}$ and $76.7 \mu \mathrm{g} /$ day, respectively [21). The cutoff for ONDST was set at $50 \mathrm{nmol} / \mathrm{L}$ (sensitivity 100\%).

\section{Statistical analysis}

Statistical Package for the Social Sciences (SPSS) version 23 (IBM, SPSS Inc, Chicago, IL) was used for the statistical analyses. The normality of data was checked using the Shapiro-Wilk method. Normally distributed data were expressed in terms of mean \pm standard deviation (SD). Non-parametric data, on the other hand, was expressed in terms of the median (interquartile range). Cortisol levels were correlated with GHQ-12 and PHQ-9 scores using Pearson/Spearman correlation (based on normality of data). Between-group comparisons were made by the Mann-Whitney $U$ test or Independent Samples $t$-test.

\section{Results}

A total of 50 patient attendants were initially recruited and subsequently screened for the presence of any of the exclusion criteria. Eleven of them met one or more of the exclusion criteria leaving behind 39 participants for final analysis (four had type 2 diabetes mellitus, two were on anti-psychotic medications, three were chronic alcohol consumers, two were obese with BMI $>28 \mathrm{~kg} / \mathrm{m} 2$ ). Amongst these 39 final participants, there were 23 males and 16 females. The mean age of the participants was $34.1 \pm 11.4$ years. The mean BMI was $21.2 \pm 1.6 \mathrm{~kg} / \mathrm{m} 2$. When the relationship of the attendants' to the index patient was considered, most of them were found to be children (28.2\%), parents (23\%), spouse (18\%), siblings (12.8\%), or others (18\% namely cousin, nephew, neighbor, son-in-law, brother-in-law). The mean duration of stay in the hospital ambience prior to being recruited in the study was $16.2 \pm 1.2$ days.

The mean GHQ-12 score of the participants was $4.8 \pm 2.3$. Among them, five participants qualified as having 'probable psychological distress', and 34 were labeled 'probable normal'. None of the participants had 'definite psychological distress'. The median PHQ-9 score of the participants was 5.0 (IQR 2.0-9.0). On the basis of the PHQ-9 score, 13 participants (33\%) had 'mild depression', five (13\%) had 'moderate depression', and three (7.6\%) had 'moderately severe depression'. All patients with a GHQ-12 score of more than seven had a PHQ-9 score of more than four. There was no statistically significant difference in either GHQ-12 or PHQ-9 scores between men and women.

\section{Cortisol dynamics among the participants}

The overall cortisol parameters of all the 39 participants have been summarized in Table 1 . 


\section{Cureus}

Cortisol Parameter

800 hours cortisol

LNPC

LNSC

24 hours UFC

Plasma cortisol after ONDST
Mean \pm SD OR Median (IQR)

$267.0 \pm 89 \mathrm{nmol} / \mathrm{L}$

$89.0 \mathrm{nmol} / \mathrm{L}$ (IQR: 48.6-166.0)

$5.0 \pm 2.1 \mathrm{nmol} / \mathrm{L}$

$31.6 \mu \mathrm{g} /$ day (IQR: 12.8-87.5)

$23.5 \mathrm{nmol} / \mathrm{L}$ (IQR: 20.2-29.1)

TABLE 1: Cortisol parameters of all the participants $(\mathrm{N}=39)$.

SD: standard deviation; IQR: interquartile range; LNPC: late-night plasma cortisol; LNSC: late-night salivary cortisol; UFC: urinary free cortisol; ONDST: overnight dexamethasone suppression test

There were no significant differences in cortisol dynamics regarding gender, although the median LNPC in women was much higher than in men $(163.5 \mathrm{nmol} / \mathrm{L}$ vs. $73.0 \mathrm{nmol} / \mathrm{L}, \mathrm{p}=0.06)$. Amongst the 39 participants, $28(72 \%)$ had a morning plasma cortisol value above the stipulated cut-off of more than $213.67 \mathrm{nmol} / \mathrm{L}$. Eighteen participants (46\%) had an LNPC above the preset cut-off of $116.04 \mathrm{nmol} / \mathrm{L}$. Seventeen of them (43.5\%) had high morning cortisol values as well. Elevated LNSC and UFC were seen in 16 (41\%) and 12 (31\%) participants, respectively. Only two individuals had elevated morning plasma cortisol, LNPC, LNSC, and UFC. None had plasma cortisol of more than $50.0 \mathrm{nmol} / \mathrm{L}$ following an ONDST.

\section{Correlation between cortisol dynamics with psychological health scores}

There was no statistically significant correlation between GHQ-12 scores and morning plasma cortisol ( $\mathrm{r}=$ $0.158, \mathrm{p}=0.338)$, LNPC ( $r s=0.077, \mathrm{p}=0.643)$, LNSC $(\mathrm{r}=-0.089, \mathrm{p}=0.558)$, UFC $(\mathrm{r} s=0.002, \mathrm{p}=0.990)$ or plasma cortisol following ONDST ( $r s=0.037, \mathrm{p}=0.824)$. Similarly, PHQ-9 scores did not show any statistically significant correlation with any of the cortisol parameters [morning plasma cortisol ( $r s=0.102, p=0.535$ ), LNPC ( $r s=0.103, p=0.531)$, LNSC ( $r s=0.150, p=0.363)$, UFC ( $r s=-0.151, p=0.360)$, plasma cortisol following ONDST ( $\mathrm{rs}=0.172, \mathrm{p}=0.296)]$.

The median GHQ-12 scores for participants with morning plasma cortisol above and below $213.67 \mathrm{nmol} / \mathrm{L}$ were 5.0 and 6.0, respectively. The corresponding PHQ-9 scores were 5.5 and 5.0, respectively. There was, however, no statistically significant difference in either of the two scores in both the sub-groups. Similarly, the median GHQ-12 scores for participants with LNPC above and below $116.04 \mathrm{nmol} / \mathrm{L}$ were 5.5 and 5.0, respectively. The corresponding PHQ-9 scores were 6.0 and 5.0, respectively. There was no statistically significant difference in either of the two scores in between the sub-groups.

The morning plasma cortisol and LNPC values were divided into quartiles. There was no statistically significant difference in GHQ-12 or PHQ-9 scores among them.

Based on PHQ-9 scores, the median morning plasma cortisol values in those with 'no depression' (score 0-4), 'mild depression' (score 5-9), and 'moderate/moderately severe depression' (score $\geqslant 10$ ) were $258.2 \mathrm{nmol} / \mathrm{L}$, $249.5 \mathrm{nmol} / \mathrm{L}$, and $338.5 \mathrm{nmol} / \mathrm{L}$, respectively. Corresponding values for LNPC were $84.1 \mathrm{nmol} / \mathrm{L}, 89.4$ $\mathrm{nmol} / \mathrm{L}$, and $166.8 \mathrm{nmol} / \mathrm{L}$ (Figure 1). 


\section{Cureus}

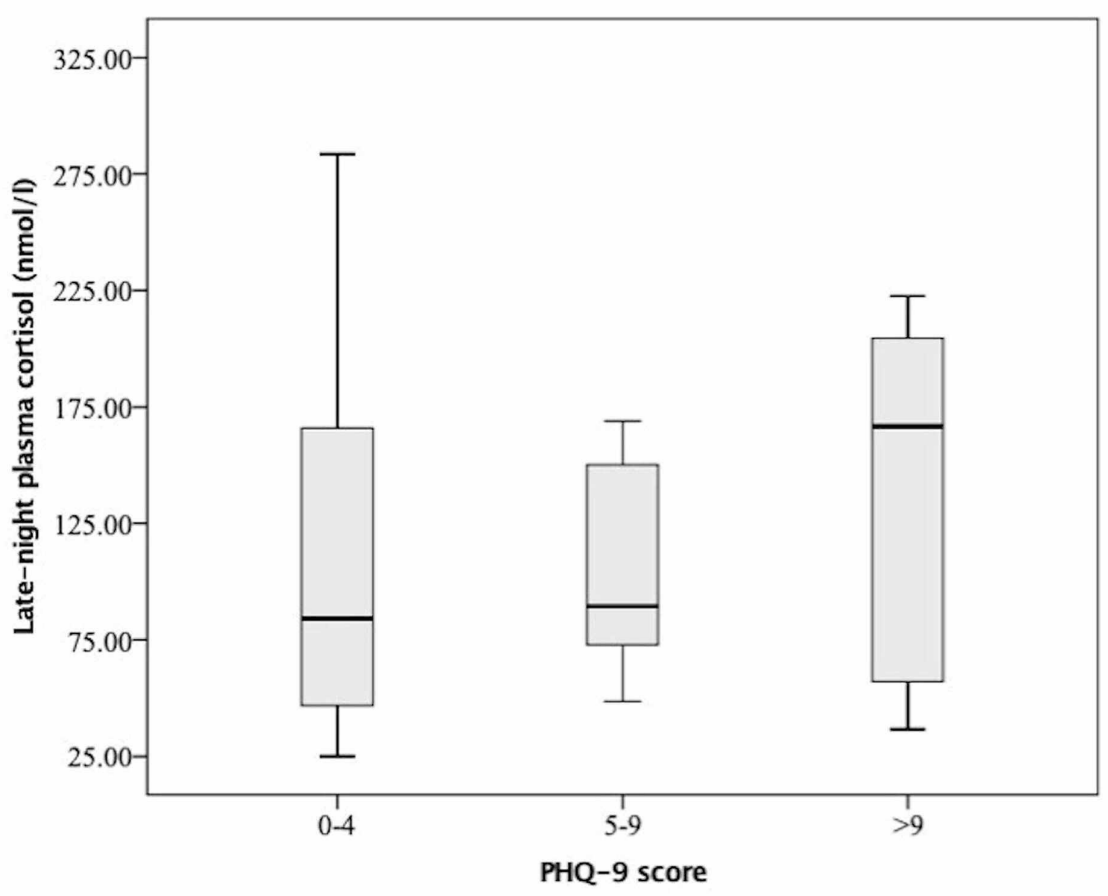

FIGURE 1: Box and whisker plot showing late-night plasma cortisol in participants with no depression (PHQ-9 score 0-4), mild depression (PHQ-9 score 5-9), and moderate/moderately severe depression (PHQ-9 score > 9).

LNSC values in the three groups were $4.4 \mathrm{nmol} / \mathrm{L}, 4.6 \mathrm{nmol} / \mathrm{L}$, and $6.0 \mathrm{nmol} / \mathrm{L}$, respectively (Figure 2).

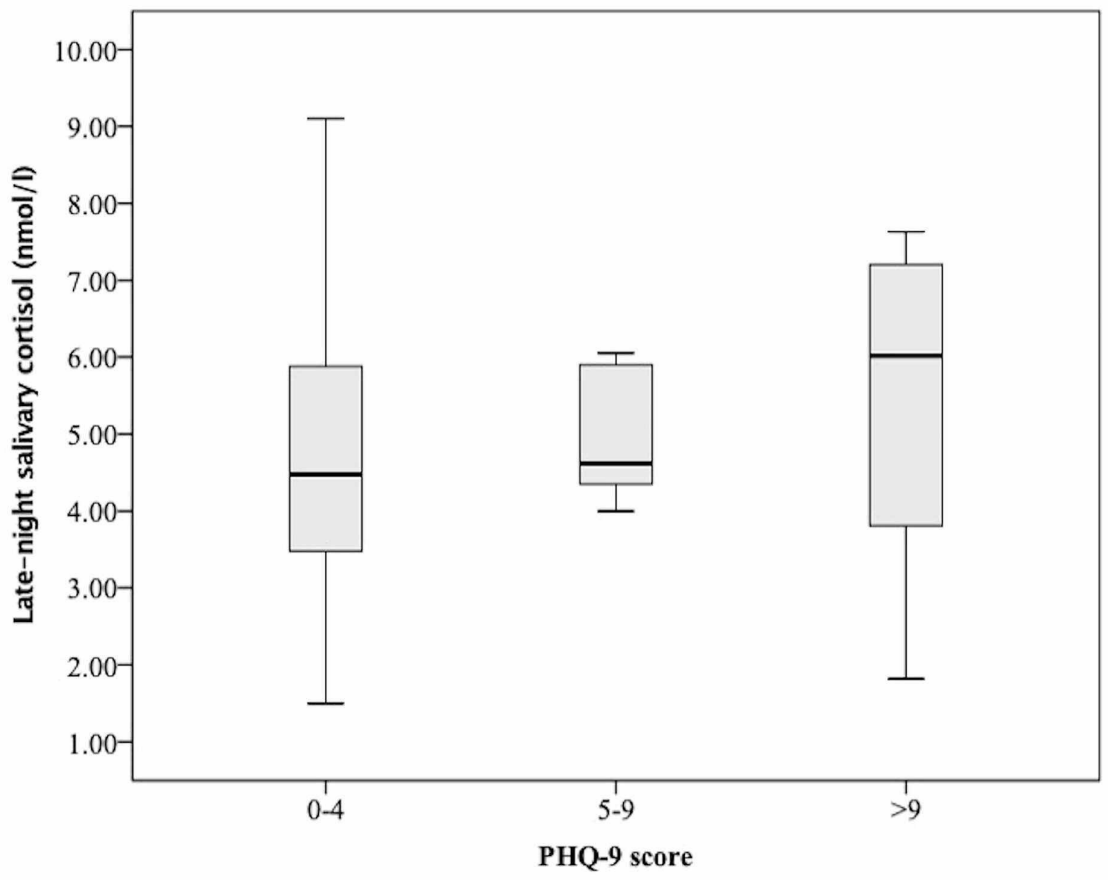

FIGURE 2: Box and whisker plot showing late-night salivary cortisol in participants with no depression (PHQ-9 score 0-4), mild depression (PHQ-9 score 5-9), and moderate/moderately severe depression (PHQ-9 score $>9$ ). 
There was no statistically significant difference amongst the three groups in morning plasma cortisol, LNPC, LNSC, UFC, or ONDST values.

Similarly, based on GHQ-12 scores, the median morning plasma cortisol values in those with 'probable normal' vs. those with 'probable psychological distress' were $254.6 \mathrm{nmol} / \mathrm{L}$ and $209.5 \mathrm{nmol} / \mathrm{L}$, respectively. Median values for LNPC were $108.0 \mathrm{nmol} / \mathrm{L}$ and $78.6 \mathrm{nmol} / \mathrm{L}$, respectively; those for LNSC were $4.8 \mathrm{nmol} / \mathrm{L}$ and $4.4 \mathrm{nmol} / \mathrm{L}$, respectively. There was no statistically significant difference among the two groups in morning plasma cortisol, LNPC, LNSC, UFC, or ONDST values.

\section{Discussion}

The above study highlights that depression is quite prevalent among attendants of patients admitted in the general medicine ward, affecting almost 55\% of the participants based on PHQ-9 score. Median morning plasma cortisol, LNPC, and LNSC were numerically higher in those with 'moderate/moderately severe depression' as compared to those with 'mild depression', however, there was no statistically significant correlation between cortisol dynamics and PHQ-9 scores.

Hospital-stay without actual hospitalization adversely affects the psychological well being contributing to stress, anxiety, depression, and maladaptive behaviors. In a study conducted among 150 patient attendants, 92.6\% and $76 \%$ of the participants had depression and anxiety, respectively [1]. Hamilton Depression Rating Scale (HDRS) was used to screen participants for depression. Instead, in our study, we used the PHQ-9 questionnaire and found that $55 \%$ of our participants had depression. Although there is a positive correlation between PHQ-9 and HDRS [22], the latter is psychometrically and conceptually flawed, as it places more emphasis on insomnia than on feelings of hopelessness, self-destructive thoughts, and suicidal ideations. Most of the scale items are poor contributors to the measurement of depression severity [23]. PHQ-9, on the other hand, explicitly addresses all the nine Diagnostic and Statistical Manual of Mental Disorders, Fourth Edition (DSM-IV) criteria for depression [24]. It has a sensitivity of 77\%, a specificity of $94 \%$, and a positive predictive value as high as $85-90 \%$ in screening for depression [25].

In a study performed amongst attendants of patients admitted in ICU, the prevalence of depression was $68 \%$ using the Hospital Anxiety and Depression Scale (HADS) [7]. This data seems to be much more realistic than that published by Rajput et al. and is concordant with our findings. The HADS, unlike the PHQ-9 scale, has the added advantage of assessing both depression (HADS-D) and anxiety (HADS-A). Although the agreement between HADS-D and PHQ-9 is moderate, studies have shown that the identified prevalence of depression is similar when using the two scales. However, as the PHQ-9 scale is derived from DSM-IV, it has a greater appeal, at least in research settings [26]. A study of 501 patients showed that PHQ was superior to HADS [24].

The other psychological health questionnaire used in our study was the GHQ-12. Only $13 \%$ of our participants were found to have 'probable psychological distress' while the rest were categorized as being 'probable normal'. This figure seems to be too low. To the best of our knowledge, GHQ-12 has hitherto never been used in the past to assess psychological distress amongst patient attendants. Our attempt at using GHQ-12 as a measure of psychological distress could have led to fallaciously low results. It is likely because of the inherent response bias on the negative items. Moreover, the PHQ-9 is known to function better than the GHQ-12 as a screening tool for depression, hence the results derived from each of the two scales are better left uncompared [27]. Large-scale observational studies comparing GHQ-12 scores of patient attendants and the general population need to be undertaken.

Our study did not find any correlation between the participants' psychological health, as measured by GHQ12/PHQ-9 and cortisol dynamics. Yet again, this was a novel attempt and was based on the premise that cortisol dynamics get altered in shift workers and nursing professionals [15-17]. However, the fact that needs to be considered in all these studies is the population being investigated. The participants in all these studies had been exposed to psychological distress for a long time instead of our volunteers who had been visiting and taking care of their patients for a mean duration of only 16.2 days. Other than subtle perturbations in the circadian rhythm of cortisol secretion, it would be too early to expect major fluctuations in cortisol dynamics over a period of two weeks. LNPC and LNSC were numerically higher in those with depression as opposed to those with no depression; in fact, those with 'moderate/moderately severe depression' had higher values than those with 'mild depression', however, there were no statistically significant differences in between these three groups likely owing to the small sample size. Rather than applying specific cut-offs to define cortisol excess, comparison of mean cortisol values of our participants with an age and sex-matched control population might have been more rewarding. In addition, existing literature supports the fact that cortisol levels are elevated in patients with major depressive disorder with little or no data in patients with mild depression [28]. As most of our participants who qualified as having depression as per the PHQ-9 scale had the milder form and only 8 participants had major depression (defined as PHQ-9 $\geqslant 10$ ), the cortisol values were not statistically different from those with no depression. Lastly, other aspects of psychological health, namely, stress, anxiety, and burnout symptoms were not assessed in the present study as they have been known to be independently associated with cortisol 
levels [16,29].

Our study does have certain limitations. Firstly, the sample size is small. However, the fact that we had planned a preliminary observational study does support our endeavor. Secondly, we do not have a control group comprising of healthy age- and sex-matched subjects residing in the community. A large communitybased survey in Southern India had shown that the prevalence of depression was only 15.1\% [30]. Hence, we can conclude that a prevalence of $55 \%$, as seen in the present study, is definitely higher than the general population. Thirdly, in comparison with a study population simultaneously recruited from a critical care setting would have provided comparative data. Lastly, cortisol dynamics were evaluated at one single time point. A serial assessment might have helped delineate the chronological alternations in cortisol dynamics.

\section{Conclusions}

Psychological health is adversely affected in attendants of patients admitted in a general hospital ward. Depression is prevalent in about half of the attendants. Cortisol dynamics, namely LNPC and LNSC, although numerically higher in depressed attendants, do not differ statistically from those without depression. A large-scale study with a properly recruited control group might prove worthwhile in unearthing the nitty-gritty of cortisol dynamics in patient attendants.

\section{Additional Information \\ Disclosures}

Human subjects: Consent was obtained by all participants in this study. Post Graduate Institute of Medical Education \& Research, Chandigarh, India issued approval NK/1664/DM/10369-70. The Institute Ethics Committee (Intramural) at their meeting has approved your above-mentioned study protocol. Animal subjects: All authors have confirmed that this study did not involve animal subjects or tissue. Conflicts of interest: In compliance with the ICMJE uniform disclosure form, all authors declare the following: Payment/services info: All authors have declared that no financial support was received from any organization for the submitted work. Financial relationships: All authors have declared that they have no financial relationships at present or within the previous three years with any organizations that might have an interest in the submitted work. Other relationships: All authors have declared that there are no other relationships or activities that could appear to have influenced the submitted work.

\section{References}

1. Rajput AH, Shaikh AR, Panhwar KA: Hospital induced psychological distress among patient attendants at Liaquat University Hospital, Jamshoro. IJEHSR. 2015, 3:21. 10.29052/IJEHSR.v3.i2.2015.21-23

2. Malhotra S, Shah R: Women and mental health in India: an overview . Indian J Psychiatry. 2015, 57:205-211. 10.4103/0019-5545.161479

3. Komachi M, Kamibeppu K: Acute stress symptoms in families of patients admitted to the intensive care unit during the first 24 hours following admission in Japan. Open J Nurs. 2015, 5:325-335. 10.4236/ojn.2015.54035

4. Zarei M, Keyvan M, Hashemizadeh H: Assessing the level of stress and anxiety in family members of patients hospitalized in the special care units. Int J Rev Life Sci. 2015, 5:118-122.

5. Ghabeesh SHA, Abu-Snieneh H, Abu-Shahror L, Abu-Sneineh F, Alhawamdeh M: Exploring the selfperceived needs for family members having adult critically ill loved person: descriptive study. Health. 2014, 6:3005-3012. 10.4236/health.2014.621338

6. Sundararajan K, Martin M, Rajagopala S, Chapman MJ: Posttraumatic stress disorder in close relatives of intensive care unit patients' evaluation (PRICE) study. Aust Crit Care. 2014, 27:183-187. 10.1016/j.aucc.2014.04.003

7. Kaur M, Dhir VB, Gulabani M, Balakrishnan I: Stress disorders in attendants of patients admitted in intensive care unit. Indian J Clin Anaesth. 2015, 2:161. 10.5958/2394-4994.2015.00020.7

8. Barth AA, Weigel BD, Dummer CD, Machado KC, Tisott TM: Stressors in the relatives of patients admitted to an intensive care unit. Rev Bras Ter Intensiva. 2016, 28:323-329. 10.5935/0103-507X.20160055

9. Karale R, Hiremath P, Mohite V, Naregal P, Karale B: The level of stress among the relatives of clients admitted in intensive care unit at tertiary care hospital - Krishna Hospital, Karad, India. Int J Health Sci Res. 2016, 6:272-276.

10. Maruiti MR, Galdeano LE, Farah OGD: Anxiety and depressions in relatives of patients admitted in intensive care units. Acta Paul Enferm. 2008, 21:636-642. 10.1590/S0103-21002008000400016

11. Heinze K, Lin A, Reniers RLEP, Wood SJ: Longer-term increased cortisol levels in young people with mental health problems. Psychiatry Res. 2016, 236:98-104. 10.1016/j.psychres.2015.12.025

12. Parker KJ, Schatzberg AF, Lyons DM: Neuroendocrine aspects of hypercortisolism in major depression . Horm Behav. 2003, 43:60-66. 10.1016/s0018-506x(02)00016-8

13. Tsigos C, Chrousos GP: Hypothalamic-pituitary-adrenal axis, neuroendocrine factors and stress . J Psychosom Res. 2002, 53:865-871. 10.1016/S0022-3999(02)00429-4

14. Fries E, Hesse J, Hellhammer J, Hellhammer DH: A new view on hypocortisolism. Psychoneuroendocrinology. 2005, 30:1010-1016. 10.1016/j.psyneuen.2005.04.006

15. Baba M, Ohkura M, Koga K, Nishiuchi K, Herrera LR, Matsuse R, Inoue T: Analysis of salivary cortisol levels to determine the association between depression level and differences in circadian rhythms of shiftworking nurses. J Occup Health. 2015, 57:237-244. 10.1539/joh.14-0079-OA

16. Marchand A, Durand P, Juster R-P, Lupien SJ: Workers' psychological distress, depression, and burnout 
symptoms: associations with diurnal cortisol profiles. Scand J Work Environ Health. 2014, 40:305-314. 10.5271/sjweh.3417

17. Yamaguti STF, Mendonça ARB de, Coelho D: Atypical secretion of cortisol in nursing professionals. [Article in Portuguese]. Rev Esc Enferm USP. 2015, 49:109-116. 10.1590/S0080-623420150000700016

18. Goldberg DP, Blackwell B: Psychiatric illness in general practice. A detailed study using a new method of case identification. Br Med J. 1970, 1:439-443. 10.1136/bmj.2.5707.439

19. Gautam S, Nijhawan M, Kamal P: Standardisation of hindi version of goldbergs general health questionnaire. Indian J Psychiatry. 1987, 29:63-66.

20. Martin A, Rief W, Klaiberg A, Braehler E: Validity of the Brief Patient Health Questionnaire Mood Scale (PHQ-9) in the general population. Gen Hosp Psychiatry. 2006, 28:71-77. 10.1016/j.genhosppsych.2005.07.003

21. Jarial KDS, Bhansali A, Mukherjee KK, et al.: Utility of a single late-night plasma cortisol and ACTH for the diagnosis of Cushing Syndrome. Endocr Pract. 2018, 24:156-162. 10.4158/EP171803

22. OR Wittkampf K, van Ravesteijn H, Baas K, et al.: The accuracy of Patient Health Questionnaire-9 in detecting depression and measuring depression severity in high-risk groups in primary care. Gen Hosp Psychiatry. 2009, 31:451-459. 10.1016/j.genhosppsych.2009.06.001

23. Bagby RM, Ryder AG, Schuller DR, Marshall MB: The Hamilton depression rating scale: has the gold standard become a lead weight?. Am J Psychiatry. 2004, 161:2163-2177. 10.1176/appi.ajp.161.12.2163

24. Löwe B, Spitzer RL, Gräfe K, et al.: Comparative validity of three screening questionnaires for DSM-IV depressive disorders and physicians' diagnoses. J Affect Disord. 2004, 78:131-140. 10.1016/s01650327(02)00237-9

25. Wittkampf KA, Naeije L, Schene AH, Huyser J, van Weert HC: Diagnostic accuracy of the mood module of the Patient Health Questionnaire: a systematic review. Gen Hosp Psychiatry. 2007, 29:388-395. 10.1016/j.genhosppsych.2007.06.004

26. Hansson M, Chotai J, Nordstöm A, Bodlund O: Comparison of two self-rating scales to detect depression: HADS and PHQ-9. Br J Gen Pract. 2009, 59:283-288. 10.3399/bjgp09X454070

27. Henkel V: Identifying depression in primary care: a comparison of different methods in a prospective cohort study. BMJ. 2003, 326:200-201. 10.1136/bmj.326.7382.200

28. Herbert J: Cortisol and depression: three questions for psychiatry . Psychol Med. 2013, 43:449-469. 10.1017/S0033291712000955

29. Pruessner JC, Hellhammer DH, Kirschbaum C: Burnout, perceived stress, and cortisol responses to awakening. Psychosom Med. 1999, 61:197-204. 10.1097/00006842-199903000-00012

30. Poongothai S, Pradeepa R, Ganesan A, Mohan V: Prevalence of depression in a large urban South Indian population - the Chennai urban rural epidemiology study (Cures - 70). Plos One. 2009, 4:7185.

10.1371/journal.pone.0007185 